\title{
Evidence for Third-Harmonic Generation in Disguise of Second-Harmonic Generation in Extreme Nonlinear Optics
}

\author{
T. Tritschler, O. D. Mücke, and M. Wegener \\ Institut für Angewandte Physik, Universität Karlsruhe (TH), Wolfgang-Gaede-Straße 1, 76131 Karlsruhe, Germany \\ U. Morgner \\ Institut für Hochfrequenztechnik und Quantenelektronik, Universität Karlsruhe (TH), \\ Engesserstraße 5, 76131 Karlsruhe, Germany \\ F. X. Kärtner \\ Department of Electrical Engineering and Computer Science and Research Laboratory of Electronics, \\ Massachusetts Institute of Technology, Cambridge, Massachusetts 02139, USA \\ (Received 22 October 2002; published 30 May 2003)
}

\begin{abstract}
In contrast with traditional nonlinear optics, a peak at the spectral position of the second harmonic of a laser can also be generated in an inversion-symmetric medium in the regime of extreme nonlinear optics. We describe the underlying mechanism of such third-harmonic generation in disguise of secondharmonic generation and compare theory with the optical as well as the radio-frequency spectra measured in recent experiments on thin $\mathrm{ZnO}$ films. The peak at twice the carrier-envelope offset frequency in the radio-frequency spectra is shown to be an unambiguous signature of such a process.

PACS numbers: 78.47.+p, 42.50.Md, 42.65.Re
\end{abstract}

Inversion symmetry has strict consequences in traditional nonlinear optics, but more relaxed ones in the regime of extreme nonlinear optics [1]. Consider an incident pulse with electric field $E(t)=\tilde{E}(t) \cos \left(\omega_{0} t+\phi\right)$. $\tilde{E}(t)$ is the envelope of the pulse, the cosine term is the carrier wave with a carrier frequency $\omega_{0}$ and a carrierenvelope offset (CEO) phase $\phi[2-4]$. Second-harmonic generation (SHG), i.e., a contribution with a carrier frequency $2 \omega_{0}$, is forbidden in an inversion-symmetric material. In traditional nonlinear optics, the spectral width of the envelope of the generated wave is much smaller than $\omega_{0}$. Thus, a peak at a spectrometer frequency $2 \omega_{0}$ cannot occur. In contrast with this, in extreme nonlinear optics, the spectral width of the envelope can approach $\omega_{0}$ or even exceed it. Equivalently, the electric field itself governs the behavior rather than the light intensity. Thus, the envelope of third-harmonic generation (THG) with carrier frequency $3 \omega_{0}$ can, e.g., lead to a low-frequency sideband (or a strong contribution) at spectrometer frequency $2 \omega_{0}$-even for an inversion-symmetric material. This phenomenon is called "THG in disguise of SHG" in what follows.

Indeed, a number of theoretical studies have dealt with this problem [5-10]. To the best of our knowledge, however, no corresponding experimental results in the regime of extreme nonlinear optics have been discussed.

How could one unambiguously distinguish THG in disguise of SHG from usual SHG? The laser pulse itself has phase $\phi$ by definition, the usual SHG has phase $2 \phi$ (even if it originates from, e.g., a $\chi^{(4)}$ process), and the third harmonic has phase $3 \phi-$ although it may exhibit a peak at spectrometer frequency $2 \omega_{0}$. Thus, the beat note of the usual SHG with the fundamental has a difference phase $\phi$, that of the THG in disguise of SHG and the fundamental has a difference phase $2 \phi$. For pulses out of a mode-locked laser oscillator, $\phi$ oscillates with the CEO frequency $f_{\phi}[2-4]$. Hence, usual SHG would lead to a beat note at frequency $f_{\phi}$ in the radio-frequency (rf) spectrum, THG in disguise of SHG to a beat note at $2 f_{\phi}$. Thus, a peak at frequency $2 f_{\phi}$ is an unambiguous experimental signature of THG in disguise of SHG.

In this Letter, we (i) describe the mechanism in more detail that can lead to THG in disguise of SHG in an inversion-symmetric medium. Next (ii), we apply this physics to a special experimental geometry, namely, to the optical and rf spectra of $\mathrm{ZnO}$, via numerical solutions of the coupled Maxwell-Bloch equations for a semiconductor. Finally in (iii), we compare these calculations directly with corresponding experiments on a $350 \mathrm{~nm}$ thin film of $\mathrm{ZnO}$ excited by intense 5 fs pulses directly from a mode-locked laser oscillator, which leads to good agreement.

(i) The mechanism.-To illustrate the general nature of the underlying physics, we start by discussing numerical solutions of the Bloch equations for an ensemble of identical two-level systems, which clearly corresponds to an inversion-symmetric medium. We do not use the rotating wave approximation; the choice of the longitudinal and transverse relaxation times $\left(T_{1}=\infty\right.$ and $\left.T_{2}=50 \mathrm{fs}\right)$ is not important unless they become comparable with the period of light. The incident pulses with a full width at half maximum (FWHM) of the intensity of $t_{\mathrm{FWHM}}=5 \mathrm{fs}$ have a $\operatorname{sinc}^{2}(t)$ shape, i.e., the electric field is given by $E(t)=\tilde{E}(t) \cos \left(\omega_{0} t+\phi\right)$ with $\tilde{E}(t)=\tilde{E}_{0} \operatorname{sinc}\left(t / t_{0}\right)$ and $\hbar \omega_{0}=1.5 \mathrm{eV}$ [11]. The peak (envelope) Rabi frequency $\Omega_{\mathrm{R}}$ is given by $\Omega_{\mathrm{R}} / \omega_{0}=0.76$, corresponding 
to the experiments described below (with a dipole matrix element $d=0.19 e \mathrm{~nm}: \Omega_{\mathrm{R}} / \omega_{0}=0.76 \Leftrightarrow \tilde{E}_{0}=$ $6 \times 10^{9} \mathrm{~V} / \mathrm{m} \Leftrightarrow$ to a peak intensity of $5 \times 10^{12} \mathrm{~W} / \mathrm{cm}^{2}$ ).

Figure 1(a) shows the square modulus of the optical polarization $P$ versus spectrometer frequency $\omega$ and versus transition frequency $\Omega$. For example, $\Omega / \omega_{0}=0.95$ on the vertical axis corresponds to the band gap of GaAs, $\Omega / \omega_{0}=2.2$ to that of $\mathrm{ZnO}$, and $\Omega / \omega_{0}=3.7$ to that of diamond. Let us, for example, consider a transition frequency $\Omega / \omega_{0}=2$, which is near the $\mathrm{ZnO}$ band gap. The corresponding cut (white curve) in Fig. 1(a) exhibits a pronounced peak at spectrometer frequency $\omega=2 \omega_{0}$
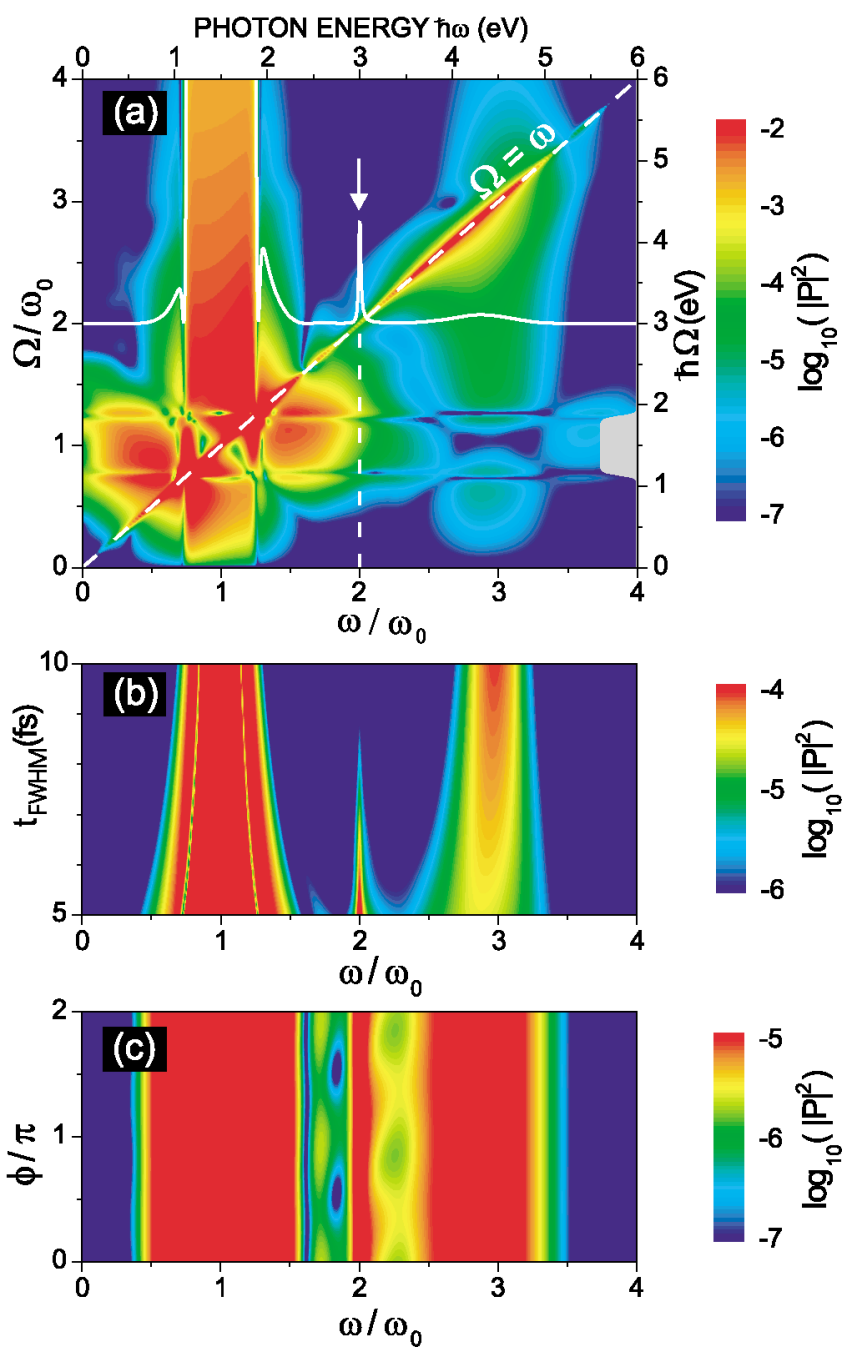

FIG. 1 (color). False-color image of the square modulus of the optical polarization $P$ (normalized) versus spectrometer frequency $\omega$ in units of the carrier frequency $\omega_{0}$ with $\hbar \omega_{0}=$ $1.5 \mathrm{eV}$. The peak Rabi frequency of the exciting $\operatorname{sinc}^{2}(t)$ pulses with duration $t_{\mathrm{FWHM}}$ is $\Omega_{\mathrm{R}} / \omega_{0}=0.76$. (a) Signal versus transition frequency $\Omega$ for a fixed CEO phase $\phi=0$ and $t_{\mathrm{FWHM}}=$ $5 \mathrm{fs}$. The white curve is a cut through the data at $\Omega / \omega_{0}=2$ (linear scale). The laser pulse spectrum is shown as the grey area on the RHS. (b) Signal versus pulse duration $t_{\mathrm{FWHM}}$ for fixed $\Omega / \omega_{0}=2$ and $\phi=0$. (c) Signal versus $\phi$ for fixed $\Omega / \omega_{0}=2$ and $t_{\mathrm{FWHM}}=5 \mathrm{fs}$. (see arrow). This contribution to the optical polarization leads to a wave at spectrometer frequency $\omega=2 \omega_{0}$, in an inversion-symmetric medium. The origin of this unusual THG in disguise of SHG is a resonance enhancement of the THG at the two-level transition frequency $\Omega$. For pulses containing many cycles of light, the laser spectrum and the usual THG contribution centered at $\omega / \omega_{0}=3$ are rather narrow and the overlap of the usual THG spectrum with the resonance at frequency $\Omega / \omega_{0}=2$ is negligible and THG in disguise of SHG does not occur. This aspect is further illustrated by the dependence on the pulse duration $t_{\mathrm{FWHM}}$ depicted in Fig. 1(b).

With increasing intensity or increasing $\Omega_{R}$, the THG in disguise of SHG contribution rises roughly with the third power of the laser intensity, which eventually overwhelms the usual SHG, which scales with the second power of the laser intensity. This resonant enhancement is clearly different from the behavior around $\Omega / \omega_{0}=1$ on the vertical axis of Fig. 1(a), which is due to carrierwave Rabi flopping, i.e., due to the large splitting of the sidebands centered around $\omega / \omega_{0}=1$ and $\omega / \omega_{0}=3$, arising from a modulation of the two-level system with the Rabi frequency as described in detail in Refs. [10,12,13].

Figure 1(c) shows the dependence on the CEO phase $\phi$ for a selected transition frequency of $\Omega / \omega_{0}=2$. All other parameters are as in Fig. 1(a). It becomes obvious that a part of the interference occurs in between the fundamental, i.e., $\omega / \omega_{0}=1$ and $\omega / \omega_{0}=2$. (In this interval one would also expect the interference of usual SHG and the fundamental.) Note that the period of the signal versus $\phi$ is $\pi$ rather than $2 \pi$, equivalent to a peak at frequency $2 f_{\phi}$ in the rf spectrum.

If one interprets the transition energy $\hbar \Omega$ in Fig. 1(a) as the band gap energy $E_{\mathrm{g}}$ of a semiconductor, the lower right-hand side (RHS) triangle formed by the $\Omega=\omega$ line experiences strong reabsorption in the semiconductor band-to-band continuum, while the upper left-hand side (LHS) triangle is in the transparency regime of the semiconductor. THG in disguise of SHG overlaps with this line.

(ii) Solutions of the coupled Maxwell-Bloch equations for $\mathrm{ZnO}$.- - In order to study corresponding reabsorption and phase-matching effects, we now present numerical solutions of the coupled Maxwell-Bloch equations in one dimension without using the rotating wave approximation and without using the slowly varying envelope approximation and accounting for the actual sample geometry of recent experiments [14]. Furthermore, for a semiconductor, one does not have a single nonlinear two-level system but rather a band continuum, i.e., one needs to integrate $P$ along the vertical axis in Fig. 1(a). The $\mathrm{ZnO}$ dipole matrix element $d=0.19 e \mathrm{~nm}$ is obtained from $k \cdot p$ perturbation theory. To be close to the experiment, we fit an ensemble of 45 two-level systems (3.3-7.9 eV) to the known measured linear dielectric function of $\mathrm{ZnO}$ over a broad 
frequency regime [15]. Thus, linear and nonlinear propagation effects as well as multiphoton absorption into high-energy states are accounted for exactly within this model. In addition to this, $\mathrm{ZnO}$ has no inversion symmetry and shows a nonvanishing $\chi^{(2)}$ susceptibility. For simplicity, we describe this aspect by a frequencyindependent second-order susceptibility, the actual value of which is taken from the literature with $\chi^{(2)}=$ $4 \times 10^{-12} \mathrm{~m} / \mathrm{V}$ [16]. Anticipating the experiments below, we model a $350 \mathrm{~nm}$ thin film of $\mathrm{ZnO}$ on a sapphire substrate [14] with dielectric constant $\epsilon_{\mathrm{s}}=(1.76)^{2}$. Furthermore, we employ excitation with a pair of collinearly propagating identical pulses with time delay $\tau$ [17]. The pulses are taken directly from the experiment [14] in the sense that the measured laser spectrum is fitted by an analytical formula [18]. Assuming unchirped laser pulses, i.e., a flat spectral phase, the electric field versus time $E(t)$ is obtained by the Fourier transform of the square root of the intensity spectrum $\propto|E(\omega)|^{2}$. The ratio of the pulse repetition frequency $f_{\mathrm{r}}$ and the CEO frequency $f_{\phi}$ is set to $f_{\mathrm{r}} / f_{\phi}=5$, with $f_{\mathrm{r}}=81 \mathrm{MHz}$.

In Fig. 2(a) we depict the calculated optical spectra versus time delay $\tau$. Note that none of the spectral components originates from the incident pulses directly; all of them are rather generated in the $350 \mathrm{~nm}$ thin $\mathrm{ZnO}$ layer. The spectral components above $530 \mathrm{~nm}$ wavelength are mainly due to self-phase modulation (SPM); those in the range from 365 to $455 \mathrm{~nm}$ wavelength are due to a combination of conventional SHG and THG in disguise of SHG. In between the two regions, interference leads to a dependence on the CEO phase $\phi$. Indeed, filtering out this region and computing the corresponding rf spectrum delivers peaks at the CEO frequency $f_{\phi}$ and at $2 f_{\phi}$ [Fig. 2(b)] as expected from our above reasoning. Interestingly, the peak at frequency $2 f_{\phi}$ is comparable in strength to that at $f_{\phi}$, equivalent to a THG in disguise of SHG contribution comparable to traditional SHG.

Before we directly compare the results of the rather complete microscopic theory to the experiment in section (iii), let us briefly show that the THG in disguise of SHG contribution described here is indeed specific for the regime of extreme nonlinear optics, i.e., that it would not occur in a perturbative off-resonant nonlinear optics approach under these conditions. To see this, let us consider the usual expansion of the nonlinear optical polarization $P_{\mathrm{NL}}(t)=\epsilon_{0} \sum_{n} \chi^{(n)} E^{n}(t)$ in terms of nonlinear optical susceptibilities $\chi^{(n)}$ up to order $N$ [9]. To allow for a direct comparison with the microscopic theory, we still solve the Maxwell equations for the actual sample geometry and for the actual laser pulses without further approximations. Going up to order $N=3$, the $\mathrm{ZnO}$ values for $\chi^{(2)}[16]$ and $\chi^{(3)}$ [19] can directly be taken from the literature. Under these conditions, the optical spectra and their dependence on $\tau$ look amazingly similar to the behavior of the microscopic approach [Fig. 2(a)]. However, within the perturbative approach, the rf spectra contain only a peak at frequency $f_{\phi}$ and no peak at $2 f_{\phi}$
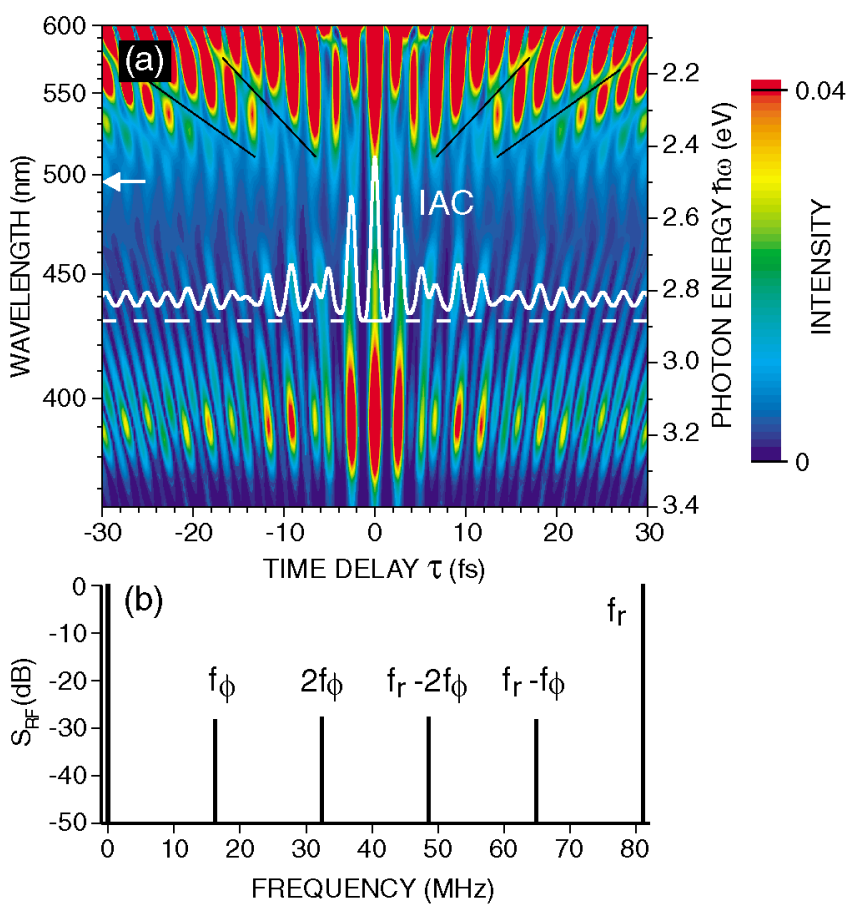

FIG. 2 (color). Theory, $\tilde{E}_{0}=6 \times 10^{9} \mathrm{~V} / \mathrm{m} \quad$ at $\tau=0$. (a) False-color image of the light intensity (normalized) emitted into the forward direction versus spectrometer photon energy $\hbar \omega$ and time delay $\tau$. The data are averaged over $\phi=$ $0 \ldots 2 \pi$. The thin black lines are a guide to the eye. The white curve labeled $I A C$ is the interferometric autocorrelation of the laser pulses. (b) Radio-frequency power spectrum $S_{\text {rf }}$ of the intensity at the spectral position indicated by the arrow on the LHS in (a), $\tau=0$.

for any position in the optical spectra. This is consistent with the intuitive expectation that no significant contribution of the third harmonic appears at the spectral position of the second harmonic in traditional nonlinear optics. This also clearly shows that rf spectra provide important information which is not obvious from the optical spectra. Including terms up to order $N=4$ or $N=$ 5 does not change the overall behavior even if $\chi^{(4)}$ and $\chi^{(5)}$ are chosen such that the corresponding contributions to the nonlinear optical polarization become comparable to the lower order ones, in which case the entire perturbative approach already becomes questionable.

(iii) Comparison with experiment.-Fig. 3 shows the experimental results corresponding to Fig. 2. In these experiments [14], 5 fs optical pulses [20] from a modelocked laser oscillator operating at $f_{\mathrm{r}}=81 \mathrm{MHz}$ repetition frequency are split into a pair of pulses with time delay $\tau$ in a balanced Michelson interferometer, stabilized by means of the Pancharatnam screw [21]. These linearly polarized pulses are focused onto a $350 \mathrm{~nm}$ thin epitaxial layer of $\mathrm{ZnO}$ on a sapphire substrate. The $\mathrm{ZnO}$ crystallographic $\vec{c}$ axis is perpendicular to the layer. Figure 3(a) has not been published so far; Fig. 3(b) corresponds to Fig. 2(b) of Ref. [14]. The agreement (compare Figs. 2 and 3) is good, especially if one keeps in 


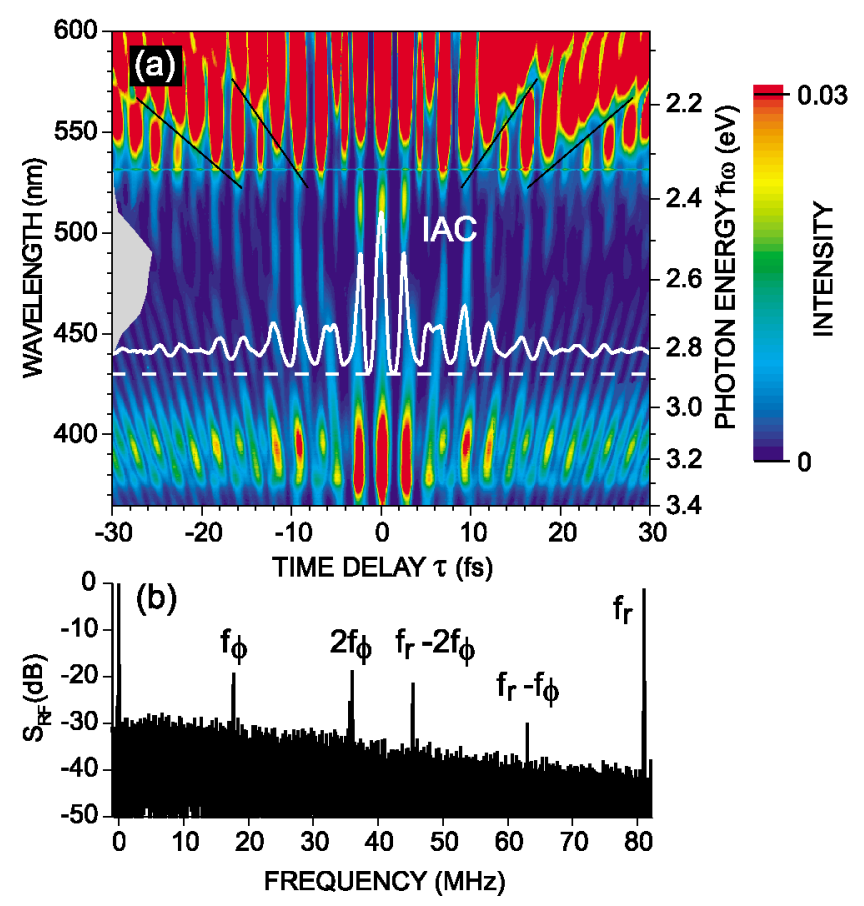

FIG. 3 (color). Experiment, $\tilde{E}_{0}=6 \times 10^{9} \mathrm{~V} / \mathrm{m}$ at $\tau=0$. (a) False-color image of the light intensity (normalized) emitted into the forward direction versus spectrometer photon energy $\hbar \omega$ and time delay $\tau$. $\phi$ is not stabilized. The thin black lines are a guide to the eye. The white curve labeled IAC is the interferometric autocorrelation of the laser pulses, obtained from an independent measurement using a $\beta$-barium borate SHG crystal. (b) Radio-frequency power spectrum $S_{\text {rf }}$ of the intensity in the spectral interval indicated by the grey area on the LHS in (a), $\tau=0$. Note the good agreement with the theory calculated under the same conditions (Fig. 2); the data in Fig. 2(a) are equidistant in photon energy, those in (a) are equidistant in wavelength.

mind that there are no fit parameters to arrange for the relative height of the SPM component, the traditional SHG and THG in disguise of SHG in the optical spectrum, or for the height of any of the peaks in the rf spectrum. In particular, note that the heights of the peaks at frequencies $f_{\phi}$ and $2 f_{\phi}$ in the rf spectrum are comparable, both for theory and experiment, indicating that THG in disguise of SHG is comparable in magnitude to conventional second-harmonic generation under these conditions. Furthermore, the nodal lines indicated by the thin black lines in Fig. 3(a) nicely match those obtained for the complete modeling [see Fig. 2(a)].

In conclusion, we have shown that a peak at twice the carrier-envelope offset frequency in the radio-frequency spectrum is an unambiguous signature of third-harmonic generation in disguise of second-harmonic generationa phenomenon, which does not occur in traditional nonlinear optics. Theory is in good agreement with experiments on a $350 \mathrm{~nm}$ thin epitaxial layer of $\mathrm{ZnO}$ excited by intense 5 fs pulses from a mode-locked laser oscillator. In general, such measurements of the CEO phase or fre- quency might also prove to be a powerful tool to identify the underlying microscopic processes in other situations of extreme nonlinear optics by providing additional information beyond the (CEO-phase-averaged) optical spectra.

The work of M.W. is supported by Projects No. DFGWe 1497/11-1 and No. DFG-We 1497/9-1, that of U. M. by Project No. DFG-Mo 850/2-1, and that of F. X. K. by ONR and NSF Grant No. ECS-0217358.

[1] T. Brabec and F. Krausz, Rev. Mod. Phys. 72, 545 (2000).

[2] A. Apolonski, A. Poppe, G. Tempea, Ch. Spielmann, Th. Udem, R. Holzwarth, T.W. Hänsch, and F. Krausz, Phys. Rev. Lett. 85, 740 (2000).

[3] D. J. Jones, S. A. Diddams, J. K. Ranka, A. Stentz, R. S. Windeler, J. L. Hall, and S. T. Cundiff, Science 288, 635 (2000).

[4] U. Morgner, R. Ell, G. Metzler, T. R. Schibli, F. X. Kärtner, J. G. Fujimoto, H. A. Haus, and E. P. Ippen, Phys. Rev. Lett. 86, 5462 (2001).

[5] R. Bavli and H. Metiu, Phys. Rev. Lett. 69, 1986 (1992).

[6] M. Yu. Ivanov, P. B. Corkum, and P. Dietrich, Laser Phys. 3, 375 (1993).

[7] A. Levinson, M. Segev, G. Almogy, and A. Yariv, Phys. Rev. A 49, R661 (1994).

[8] T. Zuo, S. Chelkowski, and A. D. Bandrauk, Phys. Rev. A 49, 3943 (1994).

[9] K. D. Moll, D. Homoelle, A. L. Gaeta, and R.W. Boyd, Phys. Rev. Lett. 88, 153901 (2002).

[10] O. D. Mücke, T. Tritschler, M. Wegener, U. Morgner, and F. X. Kärtner, Phys. Rev. Lett. 89, 127401 (2002).

[11] The long-time tails have been suppressed by a Gaussian, i.e., $\quad E(t)=\tilde{E}_{0} \operatorname{sinc}\left(t / t_{0}\right) \exp \left(-t^{2} / t_{\text {Gauss }}^{2}\right) \cos \left(\omega_{0} t+\phi\right)$ with $t_{\text {Gauss }}=28 \mathrm{fs}$ and $t_{\mathrm{FWHM}} / t_{0}=2.7831$.

[12] S. Hughes, Phys. Rev. Lett. 81, 3363 (1998).

[13] O. D. Mücke, T. Tritschler, M. Wegener, U. Morgner, and F. X. Kärtner, Phys. Rev. Lett. 87, 057401 (2001).

[14] O. D. Mücke, T. Tritschler, M. Wegener, U. Morgner, and F. X. Kärtner, Opt. Lett. 27, 2127 (2002).

[15] K. Postava, H. Sueki, M. Aoyama, T. Yamaguchi, Ch. Ino, Y. Igasaki, and M. Horie, J. Appl. Phys. 87, 7820 (2000).

[16] H. Cao, J. Y. Wu, H. C. Ong, J. Y. Dai, and R. P. H. Chang, Appl. Phys. Lett. 73, 572 (1998).

[17] J. Tate and D. Schumacher, Phys. Rev. Lett. 87, 053901 (2001).

[18] The fit formula is given by the sum of three Gaussians, i.e., by $E(\omega)=\sum_{n=1}^{3} E_{n} \exp \left[-\left(\omega-\omega_{n}\right)^{2} / \sigma_{n}^{2}\right]$, with the parameters $E_{2} / E_{1}=0.72, E_{3} / E_{1}=1.16$, and $E_{1}$ being determined by $\tilde{E}_{0} ; \hbar \omega_{1}=1.38 \mathrm{eV}, \hbar \omega_{2}=1.68 \mathrm{eV}$, and $\hbar \omega_{3}=1.82 \mathrm{eV} ; \hbar \sigma_{1}=0.10 \mathrm{eV}, \hbar \sigma_{2}=0.19 \mathrm{eV}$, and $\hbar \sigma_{3}=0.03 \mathrm{eV}$.

[19] R. Adair, L. L. Chase, and S. A. Payne, Phys. Rev. B 39, 3337 (1989)

[20] U. Morgner, F. X. Kärtner, S. H. Cho, Y. Chen, H. A. Haus, J. G. Fujimoto, E. P. Ippen, V. Scheuer, G. Angelow, and T. Tschudi, Opt. Lett. 24, 411 (1999).

[21] M. U. Wehner, M. H. Ulm, and M. Wegener, Opt. Lett. 22, 1455 (1997). 